\title{
Das geplatzte Steuerabkommen - ein schlechtes Geschäft?
}

\author{
BRIGITTE UNGER
}

$I^{2}$ den Medien hört man jüngst wieder von Politikern, wie etwa vom Finanzminister Wolfgang Schäuble, dass das vom Bundesrat abgelehnte bilaterale Steuerabkommen zwischen Deutschland und der Schweiz einen Einnahmeverlust von zehn Mrd. Euro bedeute. Diese Aussage klingt so, als sei das geplatzte Abkommen ein schlechtes Geschäft für Deutschland gewesen.

Das geplante Steuerabkommen bestand aus zwei Teilen: Für die Vergangenheit sollten die Schwarzgeldbesitzer je nach Höhe und Anlagedauer des Vermögens einmalig zwischen 21 und $41 \%$ zahlen. Für die Zukunft war eine Quellensteuer auf Kapitalerträge vorgesehen, die die Schweiz an die deutsche Steuerbehörde weiterleitet. Kontoinhaber wären anonym geblieben und die deutschen Steuerbehörden hätten nur die Möglichkeit erhalten, gezielt Kontoinformationen für eine begrenzte Anzahl von Bundesbürgern - laut ARD-Bericht 650 pro Jahr - in der Schweiz bei einem begründeten Verdacht aufSteuerhinterziehung abzufragen. Deutschland hätte sich zudem verpflichten müssen, alte Fälle von Steuerbetrug nicht mehr aufzuklären und auf die strafrechtliche Verfolgung von Mitarbeitern der Schweizer Banken wegen Beihilfe zur Steuerhinterziehung zu verzichten. Damit hätten auch Informationen von aufgekauften SteuerCDs nicht mehr verwertet werden können. Trotz Nachbesserungen wurde das Abkommen von der Bundesregierung am 17.1.2013 und vom Bundesrat am 1.2.2013 abgelehnt (BT-Drs.17/12282, BR-PlenProt Nr. 906).

War es wirklich ein schlechtes Geschäft, das Abkommen zu stoppen? Deutsche Anleger hatten nach einer 2011 in Zürich veröffentlichten Studie von Booz \& Company Ende 2010 rund 210 Mrd. Franken oder $170 \mathrm{Mrd} . €$ in der Schweiz angelegt. $102 \mathrm{Mrd}$. $€$ oder $60 \%$ der deutschen Vermögen seien jedoch am Fiskus vorbeigeschleust worden, schätzt Booz \& Company (http://www.faz.net/aktuell). Das Bedauern mancher deutscher Politiker, durch das geplatzte Abkommen auf zehn Mrd. Euro Steuereinnahmen verzichten zu müssen, klingt angesichts der Tatsache, dass doch nicht gezahlte Steuern auf $102 \mathrm{Mrd}$.€ dem deutschen Staat zustünden, befremdlich.

Die Zaghaftigkeit, mit der die Politik Steuerhinterzieher anfasst und die Tatsache, dass man bereit ist, über $100 \mathrm{Mrd}$. € Schwarzgeld mit einer gemessen an den erhofften Einnahmen um etwa ein Vierfaches höheren Steuerschuld unangetastet auf Schweizer Konten liegen zu lassen, ohne Option, diese später einfordern zu können, zeigen, was in der Finanzwissenschaft „Capturing“ heisst: die Vereinnahmung des Staates durch (finanz)wirtschaftliche Interessen. Reiche haben $102 \mathrm{Mrd}$. € lieber in schweizer Finanzmärkten angelegt, statt Wirtschaftswachstum durch zusätzliche Mittel für öffentliche Investitionen zu ermöglichen. Und der Staat war bereit, auf einen hohen zweistelligen Milliardenbetrag an zusätzlichen öffentlichen Geldern für Bildung und Umverteilung zu verzichten.

Die Ereignisse und öffentlichen Debatten rund um offshore leaks, auftauchende CDs mit Steuerinformationen, Medienberichte über Steuersünden von Prominenten, zeigen eine neue Protestbereitschaft innerhalb der zivilen Gesellschaft, die die Politik unter Zugzwang bringt. Vor allem seit der Finanzkrise, deren Umverteilungseffekte von einem Großteil der Bevölkerung als extrem ungerecht erfahren wird, weil man es versäumt hatte, die Verursacher zur Schadensbekämpfung zur Kasse zu bitten, häufen sich die zivilen Proteste.

Steueroasen innerhalb Europas beginnen langsam aufzubrechen. Die Ankündigungen Luxemburgs und Österreichs, ihr Bankgeheimnis zu lockern, sind erste positive Anzeichen.

Dieser Umschwung in der öffentlichen Auseinandersetzung erzeugt Ungewissheit und Unsicherheit innerhalb der Elite. Steuersünder werden sich nur dann freiwillig selbst anzeigen, wenn das Risiko, entdeckt zu werden, deutlich steigt. So etwa hatte der deutsche Ankauf der CDs aus Liechtenstein und der Schweiz positive Effekte in den Niederlanden. 1565 niederländische Steuerhinterzieher kehrten daraufhin heim, indem sie die "Heimkehrregelung" (inkeerregel) in Anspruch nahmen, die bei freiwilliger Deklaration Straffreiheit versprach. Im Inland setzt der niederländische Fiskus massiv auf vollkommene Information. Die Steuerbehörde weiß über alle Einkommen, Bankkonten und Finanzanlagen Bescheid. „Vergisst“ man bei der Steuererklärung, eine Anlage zu versteuern, poppt ein Kästchen „Erinnerung“von der Finanzbehörde am Computer auf, das präzise Daten über Wertpapierkonten, Spareinlagen, Hypotheken, Mieteinnahmen usw. nennt. Es findet sich auch eine Rubrik: „Besitzen Sie ein ausländisches Bankkonto?" Hierüber weiß die Finanzbehörde bis dato nichts. Wenn sie das aber auch wüsste, wäre Steuerhinterziehung innerhalb Europas kaum mehr möglich.

Zentrale Bankregister oder ein gegenseitiger perfekter Informationsaustausch würden Steuerhinterziehung innerhalb Europas unterbinden. Dies wäre sowohl ökonomisch wie auch politisch und moralisch ein deutlich besseres Geschäft als das zum Glück gescheiterte bilaterale Abkommen mit der Schweiz.

BRIGITTE UNGER, Prof. Dr., ist Wissenschaftliche Direktorin des Wirtschafts- und Sozialwissenschaftlichen Instituts (WSI) in der Hans-Böckler-Stiftung und lehrt Public Sector Economics an der Universität Utrecht.

brigitte-unger@boeckler.de 